\title{
CLINICAL RESEARCH ARTICLE Observed and calculated cerebral critical closing pressure are highly correlated in preterm infants
}

\author{
Leah I. Elizondo ${ }^{1}$, Danielle R. Rios ${ }^{1}$, Eric Vu ${ }^{2}$, Kathleen Kibler ${ }^{2}$, R. Blaine Easley ${ }^{2}$, Dean B. Andropoulos ${ }^{2}$, Sebastian Acosta ${ }^{3}$,
} Craig G. Rusin ${ }^{3}$, Jeffrey R. Kaiser ${ }^{4}$, Ken M. Brady ${ }^{5}$ and Christopher J. Rhee ${ }^{1}$

BACKGROUND: Cerebrovascular critical closing pressure (CrCP) is the arterial blood pressure (ABP) at which cerebral blood flow ceases. Preterm ABP is low and close to $\mathrm{CrCP}$. The diastolic closing margin (diastolic ABP minus $\mathrm{CrCP}$ ) has been associated with intraventricular hemorrhage in preterm infants. $\mathrm{CrCP}$ is estimated from middle cerebral artery cerebral blood flow velocity (CBFV) and $A B P$ waveforms. However, these estimations have not been validated due to a lack of gold standard. Direct observation of the $\mathrm{CrCP}$ in preterm infants with hypotension is an opportunity to validate synchronously estimated $\mathrm{CrCP}$.

METHODS: ABP and CBFV tracings were obtained from 24 extremely low birth weight infants. Recordings where diastolic CBFV was zero were identified. The gold standard CrCP was delineated using piecewise regression of ABP and CBFV values paired by rank ordering and then estimated using a published formula. The measured and estimated values were compared using linear regression and Bland-Altman analysis.

RESULTS: Linear regression showed a high degree of correlation between measured and calculated $\operatorname{CrCP}\left(r^{2}=0.93\right)$.

CONCLUSIONS: This is the first study to validate a calculated $\mathrm{CrCP}$ by comparing it to direct measurements of $\mathrm{CrCP}$ from preterm infants when $\mathrm{ABP}$ is lower than $\mathrm{CrCP}$.

Pediatric Research (2019) 86:242-246; https://doi.org/10.1038/s41390-019-0403-y

\section{INTRODUCTION}

Cerebrovascular critical closing pressure $(\mathrm{CrCP})$ is the arterial blood pressure (ABP) at which cerebral blood flow (CBF) ceases. Cerebral perfusion pressure (CPP) is commonly calculated as the difference between mean $A B P$ and the effective downstream pressure of the cerebral circulation, typically represented by intracranial pressure (ICP). However, the downstream pressure of the cerebral circulation is often higher than ICP, as ICP does not take into account the vascular wall tension, or the vascular tone. $\mathrm{CrCP}$, on the other hand, is defined to be the sum of vascular wall tension and ICP. ${ }^{1}$ In adults, CrCP has been used to estimate CPP and has been shown to correlate well with invasive measurement of CPP. ${ }^{2}$ In this way, the "effective" CPP, or closing margin, can be calculated as the difference between ABP and CrCP. The closing margin has been proposed as a metric that may be useful to delineate individualized safe ranges of $A B P$ in preterm neonates. $^{3}$

Many neonatologists routinely define hypotension as a mean $A B P$ less than gestational age (GA) in weeks; however, uncertainty remains regarding the optimal $A B P$ required to support adequate brain perfusion in preterm infants. ${ }^{4,5}$ Despite this fundamental knowledge gap, more than $80 \%$ of extremely low birth weight (ELBW) infants receive some form of treatment of hypotension in the form of a vasopressor or a fluid bolus. ${ }^{6}$ These treatments are typically given under the presumption that hypotension leads to poor perfusion of vital organs, such as the brain, and by increasing $A B P$, perfusion to vital organs can be maintained or improved.
Currently, there is no conclusive evidence that low $A B P$ is associated with poor neurological outcomes in ELBW infants compared to full-term or older children. ${ }^{7}$ Furthermore, there is no clear evidence that increasing $A B P$ results in an improvement in either short- or long-term neurological outcomes in this population. ${ }^{8,9}$ This lack of evidence may be due to an unclear definition of hypotension within this population. Alternatively, perhaps $A B P$ in isolation is not the ideal surrogate for CBF in ELBW infants.

Preterm neonates have unique features of CBF patterns when compared with term and older human subjects. Diastolic CBF velocity (CBFV) in the middle cerebral artery is strikingly low, often absent, and is always pressure passive to changes in diastolic ABP in preterm infants. ${ }^{10}$ This finding is explained by low diastolic $A B P$ in the preterm neonate that is near or even below the CrCP. Furthermore, an elevated diastolic closing margin (the difference between the diastolic $A B P$ and $\mathrm{CrCP}$ ) renders a small increase in diastolic CBFV, but is associated with severe intraventricular hemorrhage. ${ }^{3}$ Thus, the absence of CBF during diastole, a profoundly pathologic state for term neonates, is possibly a normal state for the preterm neonate. Variability in the CrCP means that CPP cannot be estimated by simple measures of $A B P$ in preterm neonates. By contrast, in term neonates or older populations without elevated ICP, diastolic $A B P$ is much greater than $\mathrm{CrCP}$, so perfusion pressure can be reasonably estimated from the ABP. Hence, the closing margin $(A B P-C r C P)$ may prove to be a useful metric to

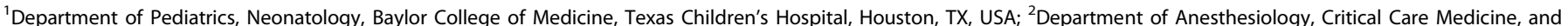

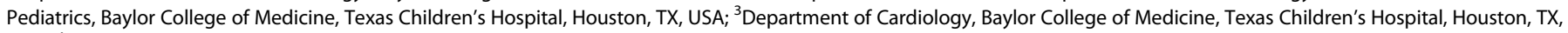

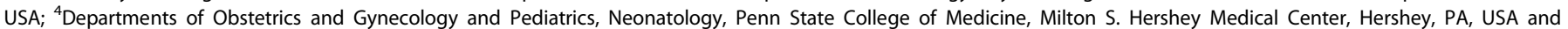
${ }^{5}$ Department of Anesthesiology, Critical Care Medicine, and Pediatrics, Northwestern University, Chicago, IL, USA

Correspondence: Christopher J. Rhee (cjrhee@texaschildrens.org)

Received: 8 November 2018 Revised: 4 April 2019 Accepted: 11 April 2019

Published online: 19 April 2019 
delineate individual targets of $A B P$ to protect the brain of the preterm neonate.

Efforts to estimate $\mathrm{CrCP}$ in preterm neonates have been confounded by the non-linear relationships between $A B P$ and $\mathrm{CBF}$ at the frequencies of the cardiac cycle. These calculations often rendered non-sensical negative values for $\mathrm{CrCP} .{ }^{11,12} \mathrm{~A}$ more recent method to estimate $\mathrm{CrCP}$ relies on the estimation of a time constant between $A B P$ and CBFV using an impedance model of the cerebrovascular bed. $^{2}$ In this model, CBFV is described by an alternating flow velocity at the frequency of the cardiac cycle, and $\mathrm{CrCP}$ is derived from an equation of impedance to flow velocity. This model has been shown to behave as predicted under conditions of altered arterial carbon dioxide tension and elevated ICP. The non-linear equations from the impedance model do not render negative values. However, true validation of calculated $\mathrm{CrCP}$ is not possible without a gold standard measure of $\mathrm{CrCP}$. In preterm neonates, when diastolic $A B P$ falls very near to, and at times below $\mathrm{CrCP}$, direct observation of $\mathrm{CrCP}$ is manifest by the absence of diastolic CBFV. This unique preterm physiology presents an opportunity to validate $\mathrm{CrCP}$ estimations from the impedance model against a gold standard measurement of CrCP. We hypothesized that the impedance-based model estimation of $\mathrm{CrCP}$ would correlate with the gold standard direct measurement of $\mathrm{CrCP}$ in preterm infants with low diastolic ABP.

\section{METHODS}

The present study used prospective data collected at Texas Children's Hospital from 2013 to 2015. Approval was obtained by the Baylor College of Medicine Institutional Review Board, and informed parental consent was obtained before enrollment. Subjects included were ELBW infants $(<1000 \mathrm{~g})$ who were mechanically ventilated and had an umbilical artery catheter in place. Those with major congenital anomalies and chromosomal abnormalities and those in extremis were excluded.

Monitoring equipment, data acquisition, and data handling All infants had measurement of ABP with an umbilical artery catheter placed for clinical monitoring. Middle cerebral artery CBFV was recorded with transcranial Doppler (TCD) ultrasound (Nicolet Vascular/Natus Medical Incorporated, San Carlos, CA, USA). Waveform data was recorded across devices using the Sickbay platform (Medical Informatics Corp, Houston, TX, USA). Digitized files were subsequently analyzed using MATLAB (MathWorks, Natick, MA, USA). A band pass filter was used to remove non-physiologic signal components such as the noise due to the respiratory cycle. Additionally, the mathematical analysis only considers the mean of the TCD and ABP, as well as the amplitude of the waveform at the frequency of the cardiac cycle. Given that the TCD and ABP are recorded from two different instruments, it is unlikely that there is common (correlated) electrical noise between the two devices. Time synchronization between the devices is accurate to approximately $6 \mathrm{~ms}$, due to the use of the Sickbay platform for synchronous data collection across physiologic devices. The amplitude of the TCD and ABP waveform at the frequency of the cardiac cycle were identified by standard Fourier methods.

\section{Observed $\mathrm{CrCP}$}

Fifty-five simultaneous recordings of middle cerebral artery CBFV with TCD and ABP from umbilical artery catheters were obtained from 24 preterm infants. Recording sessions were inspected to identify sessions where the CBFV waveform was found to be flat in the diastolic phase of the cardiac cycle. A flat diastolic flow profile indicates that there is insufficient blood pressure to generate CBF during this phase of the cardiac cycle. The pressure at which this occurs is therefore defined as the CrCP. Fourteen of the 24 infants were identified with this physiologic profile, with a median of one recording session per infant (range $1-5$ ). Each recording session was $1 \mathrm{~h}$ long and was broken into $60 \mathrm{~s}$ segments, which were then used to calculate CrCP. A median CrCP was then calculated for each recording. The sampling frequency was $120 \mathrm{~Hz}$. The amplitudes of the fundamental harmonics of $A B P$ and FV were derived using $10 \mathrm{~s}$ discrete Fourier transformations. Mean values of $A B P$ and FV were calculated in MATLAB by averaging values in a $10 \mathrm{~s}$ time window. The data were inspected to identify recordings where diastolic flow velocity was zero. ABP values were paired with corresponding CBFV as follows: each value was mapped according to the percentage of time that the cardiac cycle was below that value. Given that both the ABP and CBFV waveforms are periodic in time, a mapping can be established from one signal to another by aligning their respective oscillations. For this analysis, the cumulative distribution function (CDF) for both ABP and CBFV waveforms was calculated. Then, for each measured value of the $A B P$ waveform, the percentile of the $A B P$ waveform was found using its CDF. For instance, if CBFV equaled zero for $10 \%$ of the cardiac cycle, then the corresponding ABP was identified for the same percentage of time. The measured value of $A B P$ was then paired to the measured value of CBFV, which shared the same percentile value. The result of this data pairing operation allowed us to compare measured values of CBFV as a function of $\mathrm{ABP}$. The inflection at $\mathrm{CrCP}$ was determined from this pairing with linear piecewise regression (Fig. 1).

\section{Impedance-based $\mathrm{CrCP}$}

CrCP was calculated using the impedance-based model using the same pairings of $A B P$ and CBFV as described above for observed $\mathrm{CrCP}$. In this model, CBFV is described by an alternating flow velocity at the frequency of the cardiac cycle, and $\mathrm{CrCP}$ is derived from an equation of impedance to flow velocity. The full derivation of this method has been described in detail and validated previously. ${ }^{13,14}$ In brief, $\mathrm{CrCP}$ is estimated from the model using the following equation:

$$
\mathrm{CrCP}=\mathrm{ABP}-\frac{0.9 * \mathrm{ABP}}{\sqrt{(\mathrm{CVR} * \mathrm{Ca} * \mathrm{HR} * 2 \pi)^{2}+1}} .
$$

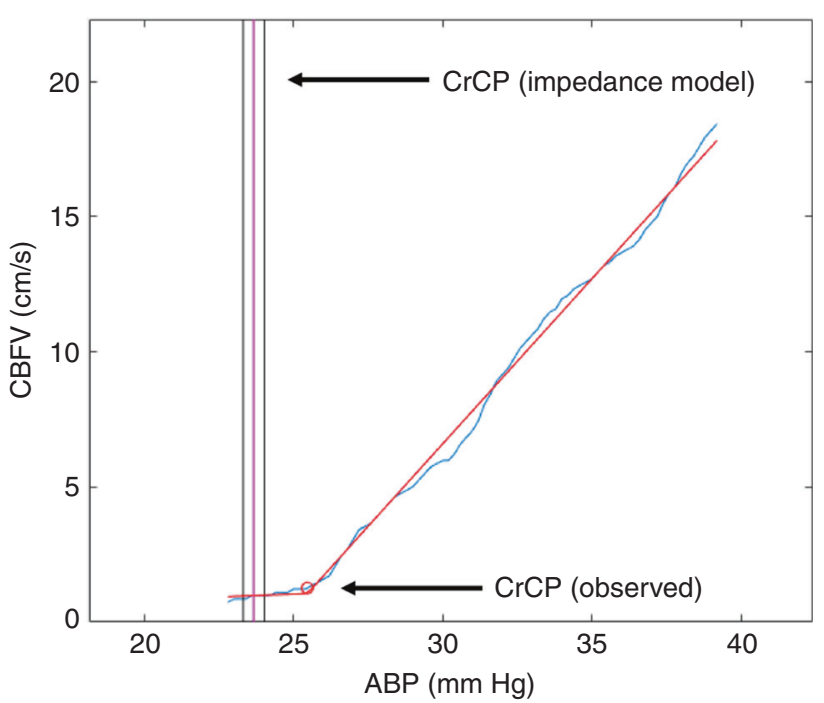

Fig. 1 Comparison of observed and calculated cerebrovascular critical closing pressure $(\mathrm{CrCP})$ in a preterm infant with low arterial blood pressure (ABP). The slanting line represents a two-line fit of the observed data with one breakpoint (observed $\mathrm{CrCP}$ ). CrCP from the impedance model is shown as a vertical line (median) with surrounding interquartile ranges 

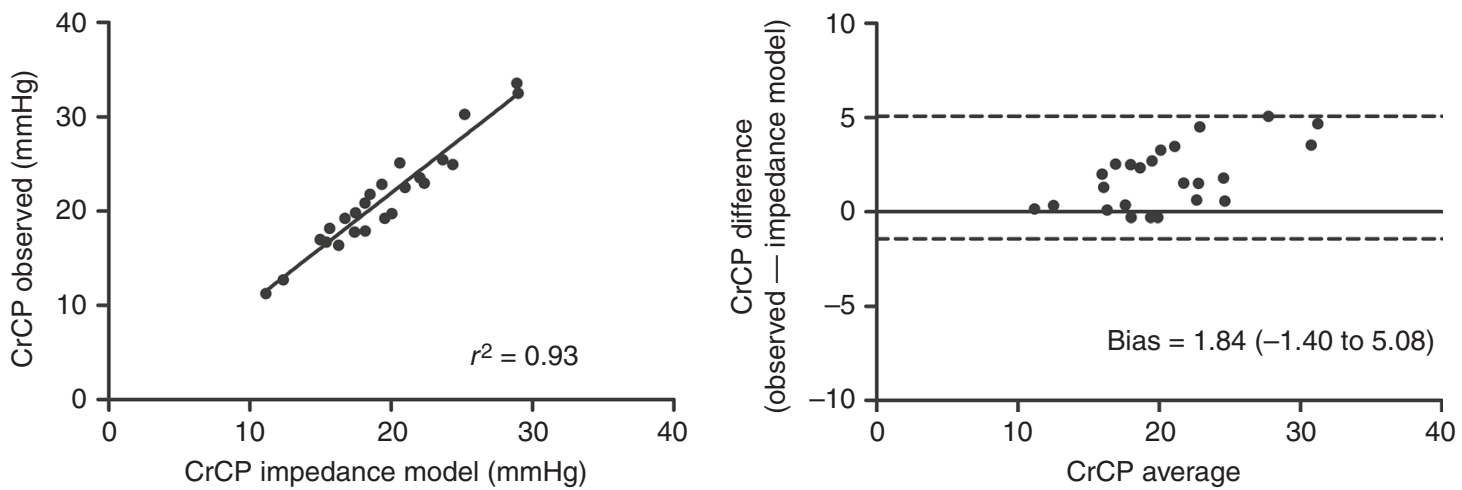

Fig. 2 a Linear regression and $\mathbf{b}$ Bland-Altman analysis of the observed cerebrovascular critical closing pressure (CrCP) versus CrCP derived using the impedance model. Dashed lines represent $95 \%$ limits of agreement

In this equation, CVR is cerebral vascular resistance and $\mathrm{Ca}$ is cerebral arterial compliance, which are not directly measureable but can be calculated using CBFV and ABP waveforms, and HR is heart rate. ${ }^{11}$ The product of resistance and compliance is termed the time constant, $\tau .^{15}$

\section{Statistics}

The continuous data (ABP and middle cerebral artery CBFV) was not tested for normality. Waveform data will not be normally distributed as both measures have physical limits at values of 0 . However, the signal processing analysis as described above does not require the data to be normally distributed. The relationship between observed and impedance-calculated $\mathrm{CrCP}$ was determined using linear regression. $\mathrm{CrCP}$ derived from the impedance model was reported as the median with interquartile ranges (IQRs). Bland-Altman analysis was performed to evaluate the bias between the mean differences. Pearson's correlation coefficient was also performed to assess the agreement between the two methods of $\mathrm{CrCP}$ estimation.

\section{RESULTS}

Infant characteristics

Infants $(n=24)$ with GA $25 \pm 0.8$ weeks (mean \pm SD; range: 23-26 weeks) and birth weight $709 \pm 141 \mathrm{~g}$ had daily 1-h recordings of $\mathrm{ABP}$ and middle cerebral artery CBFV during the first week of life. Of this cohort, 11 (46\%) were female, 23 (96\%) were exposed to antenatal steroids, 20 (83\%) were born via C-section, 3 (13\%) were from multiple gestation pregnancies, and all were mechanically ventilated during the monitoring sessions. The median Apgar scores (IQR) at 1 and 5 min were 3 (2-4) and 6 (6-7), respectively. A total of 19 (79\%) survived to hospital discharge, with all deaths occurring after the first week of life.

Cerebral critical closing pressure

The median (IQR) of the mean ABP was $27 \mathrm{~mm} \mathrm{Hg}$ (24-32) and for the mean CBFV was $10 \mathrm{~cm} / \mathrm{s}$ (8-13) in the recordings. The median directly measured $\mathrm{CrCP}$ was $20 \mathrm{~mm} \mathrm{Hg}(18-25)$. The $\mathrm{CrCP}$ estimated by the impedance-based model was determined using the same recordings (Fig. 1), with the median found to be $19 \mathrm{~mm} \mathrm{Hg}(17-22)$.

Linear regression and Bland-Altman analysis

Linear regression showed a high degree of correlation between the directly measured $\mathrm{CrCP}$ and impedance-calculated $\operatorname{CrCP}\left(r^{2}=\right.$ 0.93). The median difference between the measured $\mathrm{CrCP}$ and observed $\mathrm{CrCP}$ using the impedance model was $1.7 \mathrm{~mm} \mathrm{Hg}$ (0.3-3.1). Bland-Altman analysis showed a bias of $1.84 \mathrm{~mm} \mathrm{Hg}$ (95\% limits of agreement -1.40 to 5.08 ), with greater differences observed when CrCP was higher (Fig. 2). Pearson's correlation coefficient showed a high degree of correlation between the two methods of CrCP estimation $(r=0.96, p<0.001)$.

\section{DISCUSSION}

This is the first study to validate the use of a calculated method to estimate $\mathrm{CrCP}$ in humans. The concept that small blood vessels collapse when blood pressure falls below a critical value was first developed by Burton in 1951. ${ }^{1}$ Since that time, multiple methods to measure CrCP have been proposed and have been studied under various conditions in both animals and humans. Most of these models determine $\mathrm{CrCP}$ by assuming a linear relationship between CBFV and ABP. ${ }^{11,12}$ The major limitation of these linear models is that they produce negative, non-sensical values of $\mathrm{CrCP}$ under some conditions, such as hypercarbia and vasospasm, which cannot be interpreted physiologically. A more recent methodology developed by Baker et al. ${ }^{16}$ combines optical diffuse correlation spectroscopy measurements of pulsatile CBF in arterioles with concurrent $A B P$ data during the cardiac cycle to assess a more continuous measurement of $\mathrm{CrCP}$.

The mathematical model validated here uses the concept of impedance to $C B F$ at the oscillating $A B P$ frequency of the cardiac cycle. ${ }^{11}$ It relies on the estimation of a time constant between ABP and CBF using a model of the cerebrovascular bed with resistance and compliance in parallel. $\mathrm{CrCP}$ is calculated as a function of ABP, cerebrovascular resistance, cerebrovascular compliance, and heart rate. While cerebrovascular resistance and compliance cannot be measured, their product (the time constant of the impedance model, Tau) can be estimated from synchronous recordings of $\mathrm{ABP}$ and middle cerebral artery CBFV. ${ }^{11,17}$ The advantage of using the impedance-based method is that it renders equations that are not linear and thus does not give negative, or non-physiologic, values. ${ }^{11}$ When this multi-parameter, impedance-based model was compared to traditional calculations of $\mathrm{CrCP}$ in rabbits under three physiological conditions (increased ICP, decreased ABP, and progressive hypercapnia), the impedance-based model demonstrated positive values under each condition, whereas the traditional model produced negative values during hypercapnia. ${ }^{11}$

Furthermore, while this method continues to require measurements of $A B P$ and CBFV, the ability to calculate a CrCP by the impedance model may be more clinically advantageous as it allows for continuous and automated determination of $\mathrm{CrCP}$ at any $A B P$, not just low $A B P$, compared to the directly measured CrCP. It is also important to note that in this model, CPP is calculated as ABP minus CVP, and ICP acts as a resistor, combined with the resistor of wall tension. Therefore, changes in ICP affect Tau, which is measured, such that ICP is a contributor to the CrCP even though it is not directly measured. The consequence of this 
is that we cannot tell if ICP or vascular wall tension changed Tau, but the CrCP calculation does include an effect from ICP changes.

In this study, we measured CBFV using TCD ultrasound and inspected the data to identify times when diastolic CBFV was less than or equal to zero. $A B P$ and CBFV were paired by mapping according to the percentage of time that the cardiac cycle was below that value. Piecewise regression allowed us to determine the observed $\mathrm{CrCP}$, which we compared to the impedance-based model calculation. We found these two values to be highly correlated. The values rendered by the impedance-based model were slightly lower than the observed values, and Bland-Altman analysis also showed a slight positive bias of the observed $\mathrm{CrCP}$. While the differences were small, $<2 \mathrm{~mm} \mathrm{Hg}$, it is unclear how significant these small differences may impact the overall cerebral perfusion of these infants. When we observe a cessation of blood flow to the brain, this does not correlate with a "zero" reading on the TCD ultrasound due to the noise within the system. In the directly observed method, "zero" flow is also not affected by the angle of insonation due to the fact that surface area of the vessel, which is unknown, drops out in the equation for Tau and the confidence in this value is increased due performing repetitive measurements of $\mathrm{CrCP}$ in the cardiac cycle. ${ }^{15}$ Hence, the difference between the impedance-based model and the observed values could possibly be within the range of the noise within the TCD ultrasound.

In preterm infants, cerebral injury, such as intraventricular hemorrhage and periventricular white matter injury, is intuitively linked to instability of systemic and cerebral hemodynamics during the early post-natal period. ABP is the most commonly used clinical measurement to assess cardiovascular status and estimate the adequacy of cerebral perfusion. However, studies demonstrate that CBFV is often similar between hypotensive and normotensive preterm infants, suggesting that $A B P$ alone cannot adequately describe CBF. ${ }^{18}$ This unclear relationship between hypotension and poor neurodevelopmental outcomes highlights the importance of identifying metrics of brain perfusion that can have more relevance than $\mathrm{ABP}$. In a retrospective cohort study by Dempsey et al., ${ }^{7}$ there were no significant short-term differences between ELBW infants with permissive hypotension and good perfusion and those who were normotensive. These findings were also shown in a study by Ahn et al., ${ }^{19}$ where they demonstrated that there were no significant differences in long-term neurological outcomes as evaluated by the incidence of cerebral palsy, hearing loss, or developmental delay as assessed by Bayley scores. These studies showed worse outcomes in those infants who received treatment for hypotension; however, it is unclear whether this finding is due to the treatment itself or an underlying increased vulnerability of this treated population. Having data that is more relevant to cerebral perfusion may allow for better clinical management of the hypotensive preterm infant and eventually improve neurodevelopmental outcomes.

While $A B P$ alone is a poor surrogate for CPP, $\mathrm{CrCP}$ may be a point of zero reference for converting $A B P$ to an effective perfusion pressure. We have shown that an elevated diastolic effective perfusion pressure or closing margin (diastolic ABP minus $(\mathrm{Cr} C \mathrm{P})$ is associated with severe IVH in very low birth weight infants, whereas mean $A B P$ alone was not associated with this outcome. ${ }^{3}$ While not the primary finding in the study by Ahn et al., ${ }^{19}$ they also did not detect an association between blood pressure and severe IVH. In addition, while measured ABP does not reflect the middle cerebral artery pressure directly, it does trend to the device used for clinical decision making, which is more useful than a direct middle cerebral artery pressure measurement. In this way, the calculation of CrCP is determined from and, hence, calibrated to the clinically available number of the transduced aortic pressure. At this time, more data collection is needed to understand the significance of a low effective perfusion pressure and its possible association with white matter injury. Only by assessing this relationship will it be possible to determine if interventions can be made to modulate the effective perfusion pressure to improve short- and long-term outcomes.

One limitation of this study is that patients were selected from a cohort that was recruited for a larger, prospective study. The need for high resolution of diastolic $A B P$ and $C B F V$ resulted in some data dropout, which could have introduced unexpected bias. Another limitation is that direct measurement of $\mathrm{CrCP}$ is only possible when ABP is less than $\mathrm{CrCP}$; therefore, the impedancebased model cannot yet be validated when the closing margin (ABP minus $\mathrm{CrCP}$ ) is greater than zero using these methods. Currently, there is no "gold standard" measurement for $\mathrm{CrCP}$ and this fact allows for us to use the directly observed zero flow state as a relative "gold standard." In addition, as discussed above, our analysis showed a small, insignificant difference between the two methods, and this difference appeared to increase as CrCP increased. The clinical significance of this increase is unknown, and it is unclear if the difference between the two methods would continue to diverge as $\mathrm{CrCP}$ increases further. However, while $\mathrm{CrCP}$ does increase with increasing GA, it does also appear to plateau as infants approach term, ${ }^{13}$ so it is conceivable that this difference would also plateau and remain insignificant. Finally, it is important to note that the CrCP was calculated only by insonation of the middle cerebral artery and not smaller downstream arterioles. Differences in behavior between the macro- and microvasculature has previously been reported; ${ }^{19}$ however, this difference was not able to be evaluated in this study using TCD and warrants future investigation.

In summary, this is the first study validating the use of an impedance-based model for the determination of $\mathrm{CrCP}$ using preterm infants with diastolic ABP at or below $\mathrm{CrCP}$ as a gold standard. $\mathrm{CrCP}$ may be a more useful parameter rather than mean $A B P$ in guiding hemodynamic management of preterm infants. Additional studies are required to further evaluate the role of $\mathrm{CrCP}$ in preterm cerebral injury and whether this measure can predict both hypo- and hyper-perfusion injuries. The development of a continuous bedside monitor capable of determining $\mathrm{CrCP}$ would be a useful tool in guiding real-time management of neonatal hemodynamics with the overall goal of preventing cerebral injury.

\section{ACKNOWLEDGEMENTS}

We gratefully acknowledge the assistance of Donna Hall and the support of Baylor College of Medicine and the Texas Children's Hospital neonatologists, NICU nurses, and respiratory therapists. This work was supported by the Evie Whitlock Grant (L.I.E.) and NIH Grants K23HLI130522 (D.R.R.) 1K23NS43185 (J.R.K.), 1RO1NS060674 (J.R.K.), and K23NS091382 (C.J.R.).

\section{ADDITIONAL INFORMATION}

Competing interests: C.G.R. has a declared conflict with Medical Informatics Corp. The other authors declare no competing interests.

Publisher's note: Springer Nature remains neutral with regard to jurisdictional claims in published maps and institutional affiliations.

\section{REFERENCES}

1. Nichol, J., Girling, F., Jerrard, W., Claxton, E. B. \& Burton, A. C. Fundamental instability of the small blood vessels and critical closing pressures in vascular beds. Am. J. Physiol. 164, 330-344 (1951).

2. Varsos, G. V. et al. A noninvasive estimation of cerebral perfusion pressure using critical closing pressure. J. Neurosurg. 123, 638-648 (2015).

3. Rhee, C. J. et al. Elevated diastolic closing margin is associated with intraventricular hemorrhage in premature infants. J. Pediatr. 174, 52-56 (2016).

4. Dempsey, E. M. \& Barrington, K. J. Diagnostic criteria and therapeutic interventions for the hypotensive very low birth weight infant. J. Perinatol. 26, 677 (2006)

5. Development of audit measures and guidelines for good practice in the management of neonatal respiratory distress syndrome. Report of a Joint 
Observed and calculated cerebral critical closing pressure are highly...

LI. Elizondo et al.

246

Working Group of the British Association of Perinatal Medicine and the Research Unit of the Royal College of Physicians. Archiv. Dis. Childhood 67, 1221-1227 (1992).

6. Laughon, M. et al. Factors associated with treatment for hypotension in extremely low gestational age newborns during the first postnatal week. Pediatrics 119, 273-280 (2007).

7. Dempsey, E. M., Al Hazzani, F. \& Barrington, K. J. Permissive hypotension in the extremely low birthweight infant with signs of good perfusion. Arch. Dis. Child Fetal Neonatal Ed. 94, F241-F244 (2009).

8. Logan, J. W. et al. Early postnatal hypotension and developmental delay at 24 months of age among extremely low gestational age newborns. Arch. Dis. Child Fetal Neonatal Ed. 96, F321-F328 (2011).

9. Pellicer, A. et al. Early systemic hypotension and vasopressor support in low birth weight infants: impact on neurodevelopment. Pediatrics 123, 1369-1376 (2009).

10. Rhee, C. J. et al. The ontogeny of cerebrovascular pressure autoregulation in premature infants. J. Perinatol. 34, 926 (2014).

11. Varsos, G. V. et al. Critical closing pressure determined with a model of cerebrovascular impedance. J. Cereb. Blood Flow Metab. 33, 235-243 (2012).
12. Richards, H. K., Czosnyka, M. \& Pickard, J. D. Assessment of critical closing pressure in the cerebral circulation as a measure of cerebrovascular tone. Acta Neurochir. (Wien). 141, 1221-1227 (1999). discussion 1226-1227.

13. Rhee, C. J. et al. Ontogeny of cerebrovascular critical closing pressure. Pediatr. Res. 78, 71-75 (2015).

14. Varsos, G. V. et al. Critical closing pressure during intracranial pressure plateau waves. Neurocrit. Care 18, 341-348 (2013).

15. Kasprowicz, M. et al. Time constant of the cerebral arterial bed in normal subjects. Ultrasound Med. Biol. 38, 1129-1137 (2012).

16. Baker, W. B. et al. Noninvasive optical monitoring of critical closing pressure and arteriole compliance in human subjects. J. Cereb. Blood Flow Metab. 37, 2691-2705 (2017)

17. Kasprowicz, M. et al. Time constant of the cerebral arterial bed. Acta Neurochir. Suppl. 114, 17-21 (2012).

18. Lightburn, M. H., Gauss, C. H., Williams, D. K. \& Kaiser, J. R. Cerebral blood flow velocities in extremely low birth weight infants with hypotension and infants with normal blood pressure. J. Pediatr. 154, 824-828 (2009).

19. Ahn, S. Y. et al. Permissive hypotension in extremely low birth weight infants ( $\leq 1000$ gm). Yonsei Med. J. 53, 765-771 (2012). 